\title{
Ground states and low-temperature phases of itinerant electrons interacting with classical fields: A review of rigorous results
}

\author{
N. Macris \\ Institut de Physique théorique, EPFL, CH-1015, Lausanne, Switzerland \\ J. L. Lebowitz \\ Departments of Mathematics and Physics, Rutgers University, \\ New Brunswick, New Jersey 08903
}

(Received 2 October 1996; accepted for publication 3 December 1996)

We review, from a unified point of view, a general class of models of itinerant electrons interacting with classical fields. Applications to the static Holstein, Kondo, and Hubbard models are discussed. The ground state structure of the classical field is investigated when the electron band is half-filled. Some of the results are also valid when there is a Hubbard interaction between spin up and spin down electrons. It is found that the ground states are either homogeneous or period two Néel configurations, depending on the geometry of the lattice and on the magnetic fluxes present in the system. In the specific models, Néel configurations correspond to Peierls, magnetic or superconducting instabilities of the homogeneous state. The effect of small thermal and quantum fluctuations of the classical fields are reviewed in the context of the Holstein model. Many of the results described here originate from the work of Elliott Lieb and collaborators. (C) 1997 American Institute of Physics. [S0022-2488(97)00104-7]

\section{INTRODUCTION}

In many systems one can often distinguish degrees of freedom which have to be treated quantum mechanically from others for which a classical description is reasonable; consider e.g., the distinction between the treatment of electrons and nuclei in the Born-Oppenheimer theory of molecules. For this reason many models used in condensed matter physics contain itinerant quantum particles, usually electrons belonging to a conduction band, interacting with a classical field. We shall call these models "semi-quantum."

A much-studied model of this sort is the Falicov-Kimball model, first introduced to explain metal-insulator transitions in rare earth materials where electrons in a conduction band interact with electrons belonging to a band of localized orbitals. ${ }^{1}$ The model then consists of itinerant quantum particles interacting with "Ising spins", representing the presence or absence of a localized particle. Many exact results exist for this model; we refer to Ref. 2 for a recent review.

In this paper we analyze from a unified point of view a variety of models, namely, the static Holstein, Kondo, and (the static approximations for the repulsive and attractive) Hubbard models. ${ }^{3}$ We also review what is known rigorously when thermal and quantum fluctuations of the "classical", field are taken into account.

Let us describe briefly the physical context of these models:

The Holstein model was originally introduced to describe metal insulator transitions (Peierls instabilities) in molecular crystals. ${ }^{4}$ A breathing mode of some large molecule is singled out and modeled by Einstein oscillators coupled linearly to the electron density. The static Holstein model, obtained when the quantum fluctuations of the oscillators are neglected, has been the object of many studies related to the occurrence of charge density waves, polarons, and bipolarons in quasione-dimensional materials. ${ }^{5}$ Usually the electron band is assumed noninteracting but it is also of interest to add a Hubbard on-site interaction, between spin up and spin down electrons, in order to 
investigate the effect of electron correlations on the formation of polarons, bipolarons, and their spatial ordering.

The Kondo model concerns magnetic systems: itinerant electrons interact with magnetic impurities (quantum spins) localized at the sites of a lattice. If the localized moment of an impurity is large, it is reasonable to approximate it by a classical three-component unit vector attached to the sites of the lattice. The model obtained in this way is called the static Kondo model. (In the literature the Kondo model refers to the situation with only one magnetic impurity. Here we have in mind the so called lattice Kondo model, and a more appropriate terminology would be "static lattice Kondo model."') One can also add a Hubbard interaction term between spin up and spin down electrons.

The two other models covered by our study are closely related to the Hartree-Fock (HF) and Bardeen-Cooper-Schriefer (BCS) mean field theories of the Hubbard model. In the repulsive case one gets a model of itinerant electrons whose spin is coupled to a classical three-component vector field sitting at each lattice site. The amplitude of this vector field is variable (unlike the Kondo case where it is a unit vector). For the attractive case one finds a system of itinerant electrons interacting with a two-component vector field, whose amplitude can vary. There is an associated "elastic energy" term appearing in the Hamiltonian of both models. These models have been studied in great detail recently from a somewhat different point of view. ${ }^{6}$

In Sec. II we define a general class of models which contains all cases described above. It consists of itinerant spin up and down electrons that interact by an on-site Hubbard term and are also coupled to a classical matrix-valued field. The kinetic energy matrix of the electrons can be complex, which corresponds to the presence of an external magnetic flux. For a given configuration of the classical field one can (in principle) integrate out the quantum degrees of freedom, so that the system is reduced to a classical system with a complicated temperature and densitydependent effective energy functional for the classical field. This functional can be interpreted as the free energy of the electrons in an external potential associated with a given configuration of the classical field.

The main subject of Sec. III is to find the classical configurations that minimize this functional. We review here the solution of this problem when the electron chemical potential is adjusted so that there is an average of one electron per site, i.e., when the electron band is half-filled. The case of the static Kondo model is the simplest one. On any bipartite lattice and any magnetic flux the ground state configuration is of Neel type: this is similar to what happens in the Falicov-Kimball model. ${ }^{7,8}$ For the other models, where the amplitude as well as the direction of the vector field can vary, the situation is more complex. Indeed, depending on the geometry of the underlying lattice and the presence of an external magnetic flux, we find that the minimizing configuration is either of Néel type, or that it is homogeneous with the classical field vanishing for all sites of the lattice. In models of itinerant fermions it appears that the geometry of the lattice and the orbital coupling to a magnetic flux are important because they determine the structure of the Fermi surface of the free electron Hamiltonian, which in turn can affect the ground state structure. This will be illustrated by comparing the cases of the square and hexagonal lattices.

When the Hubbard interaction between the electrons is absent, the energy functional for a given classical configuration can be expressed in terms of a one-particle Hamiltonian, and one can then use an inequality first derived by Kennedy and Lieb in the context of the Falicov-Kimball model. ${ }^{9}$ This method breaks down when spin up and down electrons interact because, even for a given configuration of the classical field, we do not have a one-particle Hamiltonian. Nevertheless, in his treatment of the flux phase problem, Lieb showed that one can use a reflection positivity technique to get some information about the global minima of the energy functional. While this technique has been extensively used in the context of quantum and classical spin systems and bosonic systems, it was only recently extended by Lieb to models of interacting fermions. ${ }^{9}$ Lieb's ideas were extended further by Macris and Nachtergaele. ${ }^{10}$

A little studied problem is the stability of the ground states when thermal or quantum fluc- 
tuations of the classical fields are taken into account. Rigorous results, discussed in Sec. IV, have been obtained so far only for the Holstein model. For the static model, at small temperatures and large coupling, there exist at least two phases corresponding to the period two ground states on a square lattice in the half-filled band. ${ }^{7,11}$ To deal with quantum fluctuations it is convenient to integrate out the phonon degrees of freedom. Then one is left with a system of fermions interacting through a two-body potential, which is short ranged due to the quantum fluctuations. In the spinless case, and at small electron-phonon coupling, such a system belongs to the universality class of the Luttinger liquid and has been analyzed in one dimension in Ref. 12 by renormalization group methods. This analysis shows that, at least in the spinless case with small coupling, the ground state will not be ordered, for all densities.

\section{THE MODELS}

In this section we introduce a general model which is then specialized to the cases of interest. The setting is a finite lattice $\Lambda \subset \mathbf{R}^{d}$ containing $|\Lambda|$ sites. The kinetic energy of the electrons is described by a hopping matrix $T$ with elements $t_{x y}, x, y \in \Lambda$, connecting sites of $\Lambda$. Boundary conditions are either free or periodic, and are specified later. The lattice is said to be bipartite if there are two disjoint sets of sites $A$ and $B$ such that $\Lambda=A \cup B$ and $t_{x y}=0$ if $x, y \in A$ or $x, y \in B$. Examples of bipartite lattices that will be considered later are the cubical and hexagonal ones. The hopping matrix can be complex $t_{x y}=\left|t_{x y}\right| \exp \left(i \theta_{x y}\right)$, and the phase $\theta_{x y}$ has the interpretation of the line integral $\theta_{x y}=\int_{x}^{y} A . d l$, where $A$ is a vector potential associated to an external magnetic field. The sum of phases along an oriented closed circuit of the lattice is equal to the magnetic flux $\Phi_{\mathscr{C}}$ threading the circuit,

$$
\sum_{\langle x y\rangle \in \mathscr{C}} \theta_{x y}=\Phi_{\mathscr{C}}, \quad \bmod 2 \pi,
$$

where the circuit $\mathscr{C}$ is a sequence of distinct bonds $\left\langle x_{i} x_{i+1}\right\rangle$ such that $t_{x_{i} x_{i+1}} \neq 0, i=1, \ldots, k$, and $x_{k+1}=x_{1}$. We shall be using units in which $e=c=\hbar=1$.

The purely electronic contribution to the Hamiltonian is

$$
H_{\mathrm{elec}}=\sum_{x, y \in \Lambda, \sigma=\uparrow, \downarrow} t_{x y} c_{x \sigma}^{\dagger} c_{y \sigma}+U \sum_{x \in \Lambda}\left(c_{x \uparrow}^{\dagger} c_{x \uparrow}-\frac{1}{2}\right)\left(c_{x \downarrow}^{\dagger} c_{x \downarrow}-\frac{1}{2}\right),
$$

where we have included an on-site interaction of Hubbard type; $U$ can be positive or negative. When $U=0$ we set $H_{\text {elec }}=H_{\text {kin }}$.

To each site $x \in \Lambda$ we associate a $2 \times 2$ Hermitian matrix field $\Phi(x)$ with elements

$$
\Phi_{\alpha \beta}(x)=\boldsymbol{\sigma}_{\alpha \beta} \cdot \boldsymbol{\phi}(x)=\left(\begin{array}{cc}
\phi_{3}(x) & \phi_{1}(x)-i \phi_{2}(x) \\
\phi_{1}(x)+i \phi_{2}(x) & -\phi_{3}(x)
\end{array}\right),
$$

where $\alpha, \beta \in\{\uparrow, \downarrow\}, \boldsymbol{\sigma}_{\alpha \beta}=\left(\sigma_{\alpha \beta}^{1}, \sigma_{\alpha \beta}^{2}, \sigma_{\alpha \beta}^{3}\right)$ is the vector of Pauli matrices and $\boldsymbol{\phi}=\left(\phi_{1}, \phi_{2}, \phi_{3}\right)$. In the applications the matrix-valued field $\Phi(x)$ will play the role of the phonon field in the Holstein model, the impurity spin in the Kondo model, and the mean field in the Hubbard model. We note for later use the identity $\left((\boldsymbol{\sigma} \cdot \boldsymbol{\phi}(x))^{2}\right)_{\alpha \beta}=|\boldsymbol{\phi}(x)|^{2} \delta_{\alpha \beta}$. The field has an isotropic elastic energy [later on it will be convenient to view the elastic energy as a function of $|\boldsymbol{\phi}(x)|^{2}$ instead of $\left.|\boldsymbol{\phi}(x)|\right]$

$$
H_{\text {elas }}=\sum_{x \in \Lambda} P\left(|\boldsymbol{\phi}(x)|^{2}\right)
$$

where $P(y)$ is a positive convex polynomial of the form 


$$
P(y)=\sum_{j=1}^{N} a_{j}, y^{j}, \quad y \geqslant 0 .
$$

The interaction between the classical field and the electrons is on-site

$$
H_{\text {int }}=g \sum_{x \in \Lambda} \sum_{\alpha, \beta=\uparrow, \downarrow} c_{x \alpha}^{\dagger} \Phi_{\alpha \beta}(x) c_{x \beta},
$$

and the coupling constant $g$ can be positive or negative. For a given configuration $\Phi=\{\Phi(x), x \in \Lambda\}$ of the classical field the total Hamiltonian of the system is

$$
H(\Phi)=H_{\text {elec }}+H_{\text {elas }}+H_{\text {int }}
$$

The partition function is obtained by performing the trace over the electron Fock space $\mathscr{F}_{-}\left(l^{2}(\Lambda) \otimes \mathbf{C}^{2}\right)$, and by integrating over the classical field configurations $\Phi$,

$$
Z_{\Lambda}(\beta, \mu, \mathbf{h})=\int \prod_{x \in \Lambda} d \nu(\Phi(x)) \operatorname{Tr} \exp \left[-\beta\left(H(\Phi)-\mu N-\sum_{x \in \Lambda} \mathbf{h} \cdot \boldsymbol{\phi}(x)\right)\right]
$$

The average value of a local observable $A$ is given by

$$
\langle A\rangle_{\Lambda}(\beta, \mu, \mathbf{h})=\frac{1}{Z_{\Lambda}(\beta, \mu, \mathbf{h})} \int \prod_{x \in \Lambda} d \nu(\Phi(x)) \operatorname{Tr} A \exp \left[-\beta\left(H(\Phi)-\mu N-\sum_{x \in \Lambda} \mathbf{h} \cdot \boldsymbol{\phi}(x)\right)\right] .
$$

In (2.8), $N=\sum_{x, \sigma} c_{x \sigma}^{\dagger} c_{x \sigma}$ and $\mu$ and ${ }_{j} \mathbf{h}$ are chemical potentials (or external fields). The free measure $d \nu(\Phi(x))$ depends on the physical situation of interest (see later).

Since the trace in (2.8) is always positive, it is natural to set

$$
\operatorname{Tr} \exp \left[-\beta\left(H(\Phi)-\mu N-\sum_{x \in \Lambda} \mathbf{h} \cdot \boldsymbol{\phi}(x)\right)\right]=\exp [-\beta F(\Phi ; \beta, \mu, \mathbf{h})]
$$

where $F$ can be interpreted as the effective interaction energy of the classical field, induced by the itinerant electrons, or as the free energy of the electrons subjected to the external potential $\Phi(x)$. The ground state energy of a configuration $\Phi$ is defined as the zero temperature limit of $F$,

$$
E(\Phi ; \mu, \mathbf{h})=\lim _{\beta \rightarrow \infty} F(\Phi ; \beta, \mu, \mathbf{h})
$$

The global minima of the functionals $E$ and $F$ are studied in Sec. III. The appropriate space of configurations $\Phi$ over which one should minimize is determined by the choice of the free measure in $(2.8)$.

The half-filled band $\mu=0, \mathbf{h}=0$ : For a bipartite lattice, an electron-hole transformation for up and down spins,

$$
c_{x \sigma}^{\dagger} \rightarrow \epsilon_{x} c_{x \sigma}, c_{x \sigma} \rightarrow \epsilon_{x} c_{x \sigma}^{\dagger}, \quad \epsilon_{x}=1, x \in A, \quad \epsilon_{x}=-1, x \in B,
$$

transforms the Hamiltonian as $H(\Phi) \rightarrow \overline{H(-\Phi)}$, where the bar denotes complex conjugation. Hence

$$
F(\Phi ; \beta, 0,0)=F(-\Phi ; \beta, 0,0), \quad E(\Phi ; 0,0)=E(-\Phi ; 0,0) .
$$


Since under the transformation, $N \rightarrow 2|\Lambda|-N$, we find that if the free measure is invariant under $\Phi(x) \rightarrow-\Phi(x)$, then

$$
\langle N\rangle_{\Lambda}(\beta, 0,0)=\frac{1}{Z_{\Lambda}(\beta, 0,0)} \int \prod_{x \in \Lambda} d \nu(\Phi(x)) \operatorname{Tr} N \exp [-\beta H(\Phi)]=2|\Lambda|-\langle N\rangle_{\Lambda}(\beta, 0,0),
$$

so that the average number of particles $\langle N\rangle_{\Lambda}(\beta, 0,0)$ is equal to the number of sites $|\Lambda|$ for all $\beta$. For this reason the case $\mu=0, \mathbf{h}=0$ will be referred to as "the half-filled band." The results described in Secs. III and IV A are restricted to this case.

Let us now consider the special cases of (2.7) and (2.8) which are of interest to us. We start with the Kondo model for which the discussion is the simplest.

\section{A. Static Kondo model}

The static Kondo model (model A) with interacting electrons $(U \neq 0)$ is defined by

$$
H_{\mathrm{Kondo}}=H_{\mathrm{elec}}+2 g \sum_{x \in \Lambda} \mathbf{S}(x) \cdot \boldsymbol{\phi}(x),
$$

where $\mathbf{S}(x)=\frac{1}{2} \Sigma_{\alpha, \beta=\uparrow, \downarrow} c_{x \alpha}^{\dagger} \boldsymbol{\sigma}_{\alpha \beta} c_{x \beta}$ and $\boldsymbol{\phi}(x)$ is a unit vector in $\mathbf{R}^{3}$ representing an impurity spin localized at $x$. The real Kondo Hamiltonian has $\boldsymbol{\phi}(x)$ in (2.13) replaced by a quantum spin operator $\mathbf{S}_{\text {imp }}(x)$, with $\mathbf{S}_{\text {imp }}^{2}=\hbar^{2} s(s+1)$. Presumably the static Kondo model is a reasonable approximation in the semiclassical limit $\hbar \rightarrow 0, \hbar s$ fixed.

This model is a special case of (2.8) with

$$
d \nu(\Phi(x))=d \phi_{1}(x) d \phi_{2}(x) d \phi_{3}(x) \delta\left(|\phi(x)|^{2}-1\right) .
$$

The elastic term contributes only a constant so we can drop it.

The minimization of the corresponding functionals $E$ and $F$ has now to be carried out over the space

$$
\{\Phi(x), x \in \Lambda|| \boldsymbol{\phi}(x) \mid=1\} .
$$

\section{B. Static Holstein model}

The static Holstein model (model B) with interacting electrons $(U \neq 0)$ is defined by

$$
H_{\text {Holstein }}=H_{\text {elec }}+g \sum_{x \in \Lambda}\left(c_{x \uparrow}^{\dagger} c_{x \uparrow}+c_{x \downarrow}^{\dagger} c_{x \downarrow}-1\right) \phi_{3}(x)+\sum_{x \in \Lambda} P\left(\phi_{3}(x)^{2}\right)
$$

and

$$
Z_{\Lambda, \text { Holstein }}=\int \prod_{x \in \Lambda} d \phi_{3}(x) \operatorname{Tr} \exp \left[-\beta H_{\text {Holstein }}\right] .
$$

Here $\phi_{3}(x)$ represents the position of the classical oscillator attached at site $x$. In the usual Holstein model one takes $P(y)=\frac{1}{2} y$ and the oscillator is quantized so that we have to add a term

$$
-\frac{1}{2 m^{2}} \sum_{x \in \Lambda} \frac{\partial^{2}}{\partial \phi_{3}(x)^{2}}
$$

to the Hamiltonian (see Sec. IV). 
For $\Lambda$ bipartite, the coupling term in (2.16a) transforms under the electron-hole transformation $c_{x \downarrow}^{\dagger} \rightarrow \epsilon_{x} c_{x \downarrow}, c_{x \downarrow} \rightarrow \epsilon_{x} c_{x \downarrow}^{\dagger}$, on down spins only, as

$$
\left(c_{x \uparrow}^{\dagger} c_{x \uparrow}+c_{x \downarrow}^{\dagger} c_{x \downarrow}-1\right) \phi_{3}(x) \rightarrow\left(c_{x \uparrow}^{\dagger} c_{x \uparrow}-c_{x \downarrow}^{\dagger} c_{x \downarrow}\right) \phi_{3}(x)
$$

and (2.2) becomes

$$
H_{\mathrm{elec}} \rightarrow \sum_{x, y \in \Lambda} t_{x y} c_{x \uparrow}^{\dagger} c_{y \uparrow}+\sum_{x, y \in \Lambda} \overline{t_{x y}} c_{x \downarrow}^{\dagger} c_{y \downarrow}-U \sum_{x \in \Lambda}\left(c_{x \uparrow}^{\dagger} c_{x \uparrow}-\frac{1}{2}\right)\left(c_{x \downarrow}^{\dagger} c_{x \downarrow}-\frac{1}{2}\right) .
$$

Therefore for $t_{x y}=\overline{t_{x y}}$ the partition function (2.16b) is equal to (2.8a) provided $\mu=0, \mathbf{h}=0, U$ is replaced by $-U$, and

$$
d \nu(\Phi(x))=d \phi_{1}(x) d \phi_{2}(x) d \phi_{3}(x) \delta\left(\phi_{1}(x)^{2}+\phi_{2}(x)^{2}\right)
$$

Thus the static Holstein model is equivalent to the model defined by (2.8)-(2.17) as long as the lattice is bipartite and the hopping matrix elements are real.

The minimization of the corresponding functionals $E$ and $F$ has to be carried out over the space

$$
\left\{\Phi(x), x \in \Lambda \mid \phi_{1}(x)=\phi_{2}(x)=0\right\}
$$

Remark: An extended Falicov-Kimball model with interacting spin up and down electrons is obtained if in Sec. II B we take

$$
d \nu(\Phi(x))=d \phi_{1}(x) d \phi_{2}(x) d \phi_{3}(x) \delta\left(\phi_{1}(x)^{2}+\phi_{2}(x)^{2}\right) \frac{1}{2}\left[\delta\left(\phi_{3}(x)-1\right)+\delta\left(\phi_{3}(x)+1\right)\right]
$$

for the free measure. The usual Falicov-Kimball model has spinless fermions and $U=0$.

The next two models lead to a variational problem for $E$ and $F$ that has recently been studied in detail. ${ }^{6}$ We discuss them for completeness and also because the point of view presented here is somewhat different.

\section{Static approximation for the repulsive Hubbard model}

The repulsive Hubbard Hamiltonian (model C) is given by (2.2) with $U>0$. Using a path integral formalism, the partition function can be represented as that of free fermions interacting with a vector valued time-dependent auxiliary Hubbard-Stratanovich field which is coupled to the electron spin (see the Appendix and Ref. 13 for further details). The static approximation is obtained by retaining only time-independent configurations of this field. This procedure gives a model defined by (2.8) with $\mathbf{h}=0$ and,

$$
\begin{gathered}
H(\Phi)=H_{\mathrm{kin}}+H_{\mathrm{elas}}+H_{\mathrm{int}}, \quad g=\sqrt{\frac{U}{3}} \\
P\left(|\boldsymbol{\phi}(x)|^{2}\right)=\frac{1}{2}|\boldsymbol{\phi}(x)|^{2}, \\
d \nu(\Phi(x))=d \phi_{1}(x) d \phi_{2}(x) d \phi_{3}(x) .
\end{gathered}
$$

The minimization of the corresponding functionals $E$ and $F$ has to be carried out over the configuration space

$$
\left\{\Phi(x), x \in \Lambda \mid \boldsymbol{\phi}(x) \in \mathbf{R}^{3}\right\}
$$


This variational problem is equivalent to the HF mean field theory for the Hubbard model. The self-consistent equation of the mean field theory is obtained by setting the variation of $F$ with respect to the classical field equal to zero. This gives (see the Appendix)

$$
\boldsymbol{\phi}(x)=2 \sqrt{\frac{U}{3}}\langle\mathbf{S}(x)\rangle_{\Lambda}(\beta, 0,0) .
$$

To go beyond Hartree-Fock theory one needs to investigate both the thermal and quantum fluctuations around the solutions of (2.21). The model defined by (2.8) and (2.19) corresponds to taking into account only the thermal fluctuations.

\section{Static approximation for the attractive Hubbard model}

The attractive Hubbard Hamiltonian (model D) is (2.2) with $U<0$. In this case one represents the partition function with the help of a complex-valued time-dependent Hubbard-Stratanovich auxiliary field which is coupled to the electron pseudospin (see the Appendix). The static approximation is obtained by retaining only time-independent configurations of this field. This leads to (we consider only $\mu=0$ )

$$
\widetilde{Z}_{\Lambda}=\int \prod_{x \in \Lambda} d \phi_{1}(x) d \phi_{2}(x) \operatorname{Tr} \exp [-\beta \widetilde{H}]
$$

with

$$
\begin{aligned}
\widetilde{H}= & H_{\mathrm{kin}}+\sqrt{\frac{U}{2}} \sum_{x \in \Lambda}\left(c_{x \uparrow}^{\dagger} c_{x \downarrow}^{\dagger}\left(\phi_{1}(x)+i \phi_{2}(x)\right)+c_{x \downarrow} c_{x \uparrow}\left(\phi_{1}(x)-i \phi_{2}(x)\right)\right) \\
& +\frac{1}{2} \sum_{x \in \Lambda}\left(\phi_{1}(x)^{2}+\phi_{2}(x)^{2}\right) .
\end{aligned}
$$

For a bipartite lattice, making an electron-hole transformation on down spins only and putting $\phi_{1}(x) \rightarrow \epsilon_{x} \phi_{1}(x)$ and $\phi_{2}(x) \rightarrow \epsilon_{x} \phi_{2}(x)$, the coupling term in (2.22b) becomes

$$
\begin{aligned}
& c_{x \uparrow}^{\dagger} c_{x \downarrow}^{\dagger}\left(\phi_{1}(x)+i \phi_{2}(x)\right)+c_{x \downarrow} c_{x \uparrow}\left(\phi_{1}(x)-i \phi_{2}(x)\right) \rightarrow c_{x \uparrow}^{\dagger} c_{x \downarrow}\left(\phi_{1}(x)+i \phi_{2}(x)\right) \\
& +c_{x \downarrow}^{\dagger} c_{x \uparrow}\left(\phi_{1}(x)-i \phi_{2}(x)\right)
\end{aligned}
$$

and

$$
H_{\mathrm{kin}} \rightarrow \sum_{x, y \in \Lambda} t_{x y} c_{x \uparrow}^{\dagger} c_{y \uparrow}+\sum_{x, y \in \Lambda} \overline{t_{x y}} c_{x \downarrow}^{\dagger} c_{y \downarrow} .
$$

We see that for $t_{x y}=\overline{t_{x y}}$ the partition function (2.22a) is equal to (2.8), when $\mu=0, \mathbf{h}=0$, with

$$
\begin{gathered}
H(\Phi)=H_{\mathrm{kin}}+H_{\mathrm{elas}}+H_{\mathrm{int}}, \quad g=\sqrt{\frac{U}{2}} \\
P\left(|\boldsymbol{\phi}(x)|^{2}\right)=\frac{1}{2}|\boldsymbol{\phi}(x)|^{2}, \\
d \nu(\Phi(x))=d \phi_{1}(x) d \phi_{2}(x) d \phi_{3}(x) \delta\left(\phi_{3}(x)\right) .
\end{gathered}
$$

The minimization of the corresponding functionals $E$ and $F$ has to be carried out in the space 


$$
\left\{\Phi(x), x \in \Lambda \mid \phi_{3}(x)=0\right\}
$$

This variational problem is equivalent to the BCS mean field theory for the Hubbard model. The self-consistent equation of the mean field theory is obtained by setting the derivative of $F$ equal to zero. This yields (see the Appendix)

$$
\begin{aligned}
& \phi_{1}(x)-i \phi_{2}(x)=2 \sqrt{\frac{U}{2}}\left\langle c_{x \uparrow}^{\dagger} c_{x \downarrow}\right\rangle_{\Lambda}(\beta, 0,0), \\
& \phi_{1}(x)+i \phi_{2}(x)=2 \sqrt{\frac{U}{2}}\left\langle c_{x \downarrow}^{\dagger} c_{x \uparrow}\right\rangle_{\Lambda}(\beta, 0,0) .
\end{aligned}
$$

As in the repulsive case, the model (2.8)-(2.23) corresponds to taking into account only thermal fluctuations around the solutions of (2.25).

\section{MINIMIZATION OF THE ENERGY FUNCTIONALS $E$ AND $F$}

We discuss now general theorems for the structure of the global minima of the functionals (2.9) and (2.10), for the half-filled band, $\mu=0$ and $\mathbf{h}=0$. Results away from half-filling are available for the ground states of the Falicov-Kimball ${ }^{2}$ and also for the structure of the local extrema of $E$ and $F$ for the Holstein model. ${ }^{14,15}$ We set $F(\Phi ; \beta, 0,0)=F(\Phi ; \beta), E(\Phi ; 0,0)=E(\Phi)$, and $\langle-\rangle_{\Lambda}(\beta, 0,0)=\langle-\rangle_{\Lambda}(\beta)$.

A configuration $\Phi$ is called a Néel configuration if it has the form

$$
\Phi_{\alpha \beta}(x)=\epsilon_{x} w \boldsymbol{\sigma}_{\alpha \beta} \cdot \hat{n}
$$

where $\hat{n}=\left(n_{1}, n_{2}, n_{3}\right)$ is any fixed unit vector, and $w$ is a real number independent of $x$. The main result described in this section is that under appropriate conditions, in the half-filled band, the energy functionals attain their global minimum for configurations of the form (3.1). For the static Kondo model we necessarily have $w=1$. For the other models it is evident that, when the coupling between electrons and classical field $g=0$, the homogeneous configuration corresponding to $w=0$ is a ground state. When $g \neq 0$ this state may remain stable, or become unstable so that $w$ acquires a nonzero value depending on $\beta, g$, and $U$. In the context of the Holstein model, $w \neq 0$ is the so-called Peierls instability, while for the static approximations to the attractive and repulsive Hubbard models, $w \neq 0$ means respectively that there is a superconducting or magnetic instability.

\section{A. Interacting electrons, $\boldsymbol{U} \neq \mathbf{0}$}

For the Hamiltonian (2.7) with $U \neq 0$ it turns out that one can adapt the reflection positivity techniques, previously used for quantum spin or bosonic systems, ${ }^{16,17}$ as was first shown by Lieb for the Hubbard model. ${ }^{9}$

Some restrictions on the geometry of the lattice are needed, and instead of formulating the most general result we consider here three representative examples. These are: (a) onedimensional rings with an even number of sites, (b) the bipartite square (or cubic) lattice with periodic boundary conditions, and (c) the bipartite hexagonal lattice with periodic boundary conditions. Note that for all these cases the number of sites contained in any closed loop is even. For such lattices $\Lambda$ embedded in $\mathbf{R}^{d}$, consider a $(d-1)$-dimensional hyperplane $P$ not containing any vertex of $\Lambda$, separating $\Lambda$ in two sets of vertices called $L$ ("left") and $R$ ("right"), so that $\Lambda=L \cup R$. When $L$ and $R$ are related to each other by a geometric reflection across $P$, we say that $P$ is a reflection plane for $\Lambda$. For example, for the bipartite square lattice with periodic boundary conditions, all planes perpendicular to the two coordinate axis and not containing any vertex are reflection planes. 
Given $\left\{t_{x y}\right\}, t_{x y}=\left|t_{x y}\right| \exp \left(i \theta_{x y}\right)$, the flux configuration is called a canonical flux configuration if (a) for one-dimensional rings,

$$
\sum_{\langle x y\rangle \in \Lambda} \theta_{x y}=\pi\left(\frac{|\Lambda|}{2}-1\right), \quad \bmod 2 \pi,
$$

(b) for the bipartite square lattice on a torus,

$$
\sum_{\langle x y\rangle \in F} \theta_{x y}=\pi, \quad \bmod 2 \pi,
$$

for all elementary square plaquettes $F$, and

$$
\sum_{\langle x y\rangle \in \Lambda_{i}} \theta_{x y}=\pi\left(\frac{\left|\Lambda_{i}\right|}{2}-1\right), \quad \bmod 2 \pi, \quad i=1,2,
$$

$\Lambda_{i}, i=1,2$, the two nontrivial loops of the torus along the coordinate axis; and (c) for the bipartite hexagonal lattice on a torus,

$$
\sum_{\langle x y\rangle \in F} \theta_{x y}=0, \quad \bmod 2 \pi,
$$

for all elementary hexagons $F$, and

$$
\sum_{\langle x y\rangle \in \Lambda_{i}} \theta_{x y}=\pi\left(\frac{\left|\Lambda_{i}\right|}{2}-1\right), \quad \bmod 2 \pi, \quad i=1,2,
$$

$\Lambda_{i}, i=1,2$, the two nontrivial loops of the torus along the coordinate axis.

For example a flux which is uniformly equal to zero (realized by taking all $\theta_{x y}=0$ ) is non canonical for a cubical lattice, while it is canonical for the hexagonal one. We note that in all the above cases it is possible to choose a gauge (i.e., a choice of phases $\left\{\theta_{x y}\right\}$ ) such that all $t_{x y}$ are real. The following theorem ensures that under appropriate conditions the global minimum of $E$ and $F$ is attained among Néel configurations.

Theorem 1: Let $\Lambda$ be one of the lattices (a), (b), and (c). Suppose that the flux configuration is canonical and that the moduli $\left\{\left|t_{x y}\right|\right\}$ are invariant under geometric reflections through all reflection planes $P$ of $\Lambda$. Then there exists at least one minimizer of $E(\Phi)$ and $F(\Phi ; \beta)$ which is a Néel configuration (3.1).

Remarks: (i) To apply the theorem to the four specific models described in Sec. II, one has to specify the space over which the minimization is carried out. For the static Kondo model this space is (2.15) so that the minimizer has $w=1$ in (3.1). For the static Holstein and attractive Hubbard models the spaces are respectively (2.18) and (2.24) so that the minimizers have respectively $\hat{n}=(0,0,1)$ and $\hat{n}=\left(n_{1}, n_{2}, 0\right)$. For the repulsive Hubbard model there is no constraint.

(ii) We do not know of any general statement about the unicity of the minimum. All we know is that there is at least one minimum of the form (3.1), and if there is another one, we cannot exclude that it is outside the class of Néel configurations.

(iii) Except for the case of the Kondo model A where the ground state is completely determined by Theorem 1, the value of $w$ will, in general, depend on $\beta, g, U$ and $\left\{\left|t_{x y}\right|\right\}$. When $U=0$, $w$ can be found explicitly in principle, because the Hamiltonian reduces to that of free electrons in a period two potential (see Sec. III B).

(iv) A straightforward application of reflection positivity also implies that given a reflection plane $P$, then if $r(x)$ is the reflection of a site $x$ through $P$, 


$$
\langle\boldsymbol{\phi}(x) \cdot \boldsymbol{\phi}(r(x))\rangle_{\Lambda}(\beta) \leqslant 0
$$

at any finite temperature. This inequality means that there is a tendency towards antiferromagnetic behavior but does not imply that there is long range order. Indeed it is valid for the onedimensional ring, where one does not expect long range order for any finite temperature.

A similar result for the case of the extended Falicov-Kimball model mentioned, after Sec. II B was pointed out in Ref. 10. The proof given below is based on the methods of Ref. 9 and 10.

Sketch of the Proof. Given a reflection plane $P$ we introduce new creation and annihilation operators defined for all $x \in \Lambda=L \cup R$,

$$
d_{x \sigma}^{\dagger}=c_{x \sigma}^{\dagger} e^{i \pi N_{L}}, \quad d_{x \sigma}=e^{i \pi N_{L}} c_{x \sigma}
$$

where $N_{L}=\Sigma_{x \in L, \sigma=\uparrow, \downarrow} c_{x \sigma}^{\dagger} c_{x \sigma}$. The new operators commute if $x \in L, y \in R$ or if $x \in R, y \in L$, and satisfy the canonical anticommutation relations if $x \in L, y \in L$ or if $x \in R, y \in R$. In terms of the new operators the Hamiltonian (2.7) takes the same form with the $c$ s replaced by the $d \mathrm{~s}$. It can be decomposed as

$$
H(\Phi)=H_{L}\left(\Phi_{L}\right)+H_{R}\left(\Phi_{R}\right)+H_{1}
$$

with

$$
\begin{gathered}
H_{L / R}\left(\Phi_{L / R}\right)= \\
\sum_{x, y \in L / R} t_{x y} d_{x \sigma}^{\dagger} d_{y \sigma}+U \sum_{x \in L / R}\left(d_{x \uparrow}^{\dagger} d_{x \uparrow}-\frac{1}{2}\right)\left(d_{x \downarrow}^{\dagger} d_{x \downarrow}-\frac{1}{2}\right) \\
+g \sum_{x \in L / R} \sum_{\alpha, \beta=\uparrow ; \downarrow} d_{x \alpha}^{\dagger} \Phi_{\alpha \beta}(x) d_{x \beta}+\sum_{x \in L / R} P\left(|\boldsymbol{\phi}(x)|^{2}\right), \\
H_{1}=\sum_{x \in L, y \in R} t_{x y} d_{x \sigma}^{\dagger} d_{y \sigma}+\sum_{x \in R, y \in L} t_{x y} d_{x \sigma}^{\dagger} d_{y \sigma}
\end{gathered}
$$

and $\Phi_{L / R}=\{\Phi(x), x \in L / R\}$. Because the flux configuration is canonical, it is always possible to choose a gauge such that $\left\{t_{x y}\right\}$ is real and moreover $t_{u v}=-\left|t_{u v}\right|$ for the bonds $\langle u v\rangle$ that are intersected by the reflection plane $P$. Then, performing an electron-hole transformation for the sites $x \in R$ only,

$$
\begin{gathered}
H_{L}\left(\Phi_{L}\right) \rightarrow H_{L}\left(\Phi_{L}\right), \\
H_{R}\left(\Phi_{R}\right) \rightarrow H_{R}\left(-\Phi_{R}\right), \\
H_{1} \rightarrow \widetilde{H}_{1}=-\sum_{x \in L, y \in R}\left|t_{x y}\right| d_{x \sigma}^{\dagger} d_{y \sigma}^{\dagger}-\sum_{x \in R, y \in L}\left|t_{x y}\right| d_{x \sigma} d_{y \sigma} .
\end{gathered}
$$

The transformed Hamiltonian $\widetilde{H}(\Phi)$ obtained from (3.7) is given by the sum of the three terms on the rhs of (3.10):

$$
\widetilde{H}(\Phi)=H_{L}\left(\Phi_{L}\right)+H_{R}\left(-\Phi_{R}\right)+\widetilde{H}_{1}
$$

For $\mu=\mathbf{h}=0$, the trace in (2.9) is invariant under all the transformations performed above, so that $H(\Phi)$ and $\widetilde{H}(\Phi)$ have the same ground state and effective energies $E(\Phi)$ and $F(\Phi ; \beta)$. The Hamiltonian (3.11) is reflection positive so that a direct application of the Dyson-Lieb-Simon inequality $^{16}$ or its ground state version ${ }^{18}$ implies that at least one of the two Hamiltonians, 


$$
\begin{gathered}
H_{L}\left(\Phi_{L}\right)+H_{R}\left(\Phi_{L}^{r}\right)+\widetilde{H}_{1}, \\
H_{L}\left(-\Phi_{R}^{r}\right)+H_{R}\left(-\Phi_{R}\right)+\widetilde{H}_{1},
\end{gathered}
$$

has a lower energy, where $\Phi_{L / R}^{r}$ is the configuration obtained by reflecting $\Phi_{L / R}$ across $P$. Therefore given a configuration $\Phi$ and a reflection plane $P$ separating $\Phi_{L}$ and $\Phi_{R}, E(\Phi)$ and $F(\Phi ; \beta)$ are lowered when $\Phi$ is replaced by one of the two new configurations formed by $\Phi_{L}$ and $-\Phi_{L}^{r}$ or by $-\Phi_{R}^{r}$ and $\Phi_{R}$. Iterating this inequality with respect to all reflection planes yields a lower bound which is attained for a configuration of Néel type (3.1).

\section{B. Noninteracting electrons, $U=0$}

For $U=0$, the Hamiltonian $H_{\text {kin }}+H_{\text {int }}$ in (2.7) is the second quantized form of a one-particle Hamiltonian

$$
h_{x y, \alpha \beta}=t_{x y} \delta_{\alpha \beta}+g \Phi_{\alpha \beta}(x) \delta_{x y},
$$

$x, y \in \Lambda, \alpha, \beta \in\{\uparrow, \downarrow\}$ which acts on wave functions in $l^{2}(|\Lambda|) \otimes \mathbf{C}^{2}$. The trace over the electron Fock space in (2.9) can be performed and for $\mu=\mathbf{h}=0$ this leads to

$$
F(\Phi ; \beta)=-\frac{1}{\beta} \operatorname{tr} \ln \cosh \frac{\beta}{2} \sqrt{h^{2}}+\sum_{x \in \Lambda} P\left(|\boldsymbol{\phi}(x)|^{2}\right),
$$

and by taking the limit $\beta \rightarrow \infty$

$$
E(\Phi)=-\frac{1}{2} \operatorname{tr} \sqrt{h^{2}}+\sum_{x \in \Lambda} P\left(|\boldsymbol{\phi}(x)|^{2}\right) .
$$

In (3.14) and (3.15) the trace is on the space $l^{2}(|\Lambda|) \otimes \mathbf{C}^{2}$.

The minimization of functionals of the type (3.14) and (3.15) was first achieved, in the case of the Falicov-Kimball model, by Kennedy and Lieb ${ }^{7}$ for zero magnetic flux (on general bipartite graphs) and by Lieb and Loss ${ }^{8}$ when the flux is present. The method presented below for flux configurations which are canonical relies on an application of Theorem 1, and is different than in Ref. 8. However, it breaks down if the flux is noncanonical, so that in this respect the methods of Ref. 7 and 8 are more general.

Case of canonical flux configuration: Application of Theorem 1. In this paragraph we consider the problem of determining the value of $w$ in (3.1) for the models B-D. When the lattice and the set of $\left\{t_{x y}\right\}$ satisfy the hypothesis in Theorem 1, we know that a minimum of (3.14) and (3.15) is attained in the class of Néel configurations (3.1). For such configurations we have

$$
h^{2}=T \otimes \mathbf{1}_{2}+g^{2} w^{2} \mathbf{1}_{\Lambda} \otimes \mathbf{1}_{2},
$$

where $\mathbf{1}_{n}$ is the $n \times n$ identity matrix. Substituting (3.16) in (3.14) and (3.15) we see that the problem is reduced to the minimization of functions of one variable $w^{2}$,

$$
\begin{gathered}
f\left(w^{2}, \beta\right)=-\frac{2}{\beta} \operatorname{tr} \ln \cosh \frac{\beta}{2} \sqrt{T^{2}+g^{2} w^{2} \mathbf{1}_{\Lambda}}+|\Lambda| P\left(w^{2}\right), \\
e\left(w^{2}\right)=-\operatorname{tr} \sqrt{T^{2}+g^{2} w^{2} \mathbf{1}_{\Lambda}}+|\Lambda| P\left(w^{2}\right) .
\end{gathered}
$$

In (3.17) and (3.18) the trace over the spin degree of freedom has been performed and the remaining one is over the space $l^{2}(\Lambda)$. 
Since $f$ is a convex function of $w^{2}$, it has a unique minimizer, say $w_{0}^{2}$ It follows that $\Phi_{0}(x)=\epsilon_{x} w_{0} \boldsymbol{\sigma} \cdot \hat{n}$ is a minimizer for $F(\Phi ; \beta)$ (however, we cannot conclude that $\Phi_{0}$ is unique). To compute $w_{0}$ we note that since $P\left(w^{2}\right)$ grows at least as $w^{2}$ and the square root behaves as $w$ for $w^{2} \rightarrow \infty$, the only possibilities for the minimum of (3.17) are:

(i) $w_{0}^{2}$ is a solution of the equation $f^{\prime}\left(w_{0}^{2}, \beta\right)=0$, i.e.,

$$
P^{\prime}\left(w_{0}^{2}\right)=\frac{g^{2}}{2|\Lambda|} \operatorname{tr}\left[\left(T^{2}+g^{2} w_{0}^{2} \mathbf{1}_{\Lambda}\right)^{-1 / 2} \tanh \frac{\beta}{2} \sqrt{T^{2}+g^{2} w_{0}^{2} \mathbf{1}_{\Lambda}}\right]
$$

if it exists.

(ii) If (3.19) has no solution, then the minimum of $f$ is attained at the boundary of the domain of $w^{2}$, i.e., for $w_{0}=0$.

For the minimum of (3.18) the situation is analogous, with (3.19) replaced by $e^{\prime}\left(w_{0}^{2}\right)=0$, i.e.,

$$
P^{\prime}\left(w_{0}^{2}\right)=\frac{g^{2}}{2} \operatorname{tr}\left[\left(T^{2}+g^{2} w_{0}^{2} \mathbf{1}_{\Lambda}\right)^{-1 / 2}\right]
$$

Let us now discuss the properties of Eqs. (3.19) and (3.20). We begin with the case of finite temperatures. If $P$ has a harmonic term, $a_{1}>0$, then for $\beta g^{2}<2 a_{1}$ the minimizer is $w_{0}=0$. Indeed since $P$ is convex, $P^{\prime}$ grows, so $P^{\prime}\left(w^{2}\right) \geqslant a_{1}$. Moreover, using $\tanh x \leqslant x$ we have that

$$
\frac{g^{2}}{2|\Lambda|} \operatorname{tr}\left[\left(T^{2}+g^{2} w^{2} \mathbf{1}_{\Lambda}\right)^{-1 / 2} \tanh \frac{\beta}{2} \sqrt{T^{2}+g^{2} w^{2} \mathbf{1}_{\Lambda}}\right] \leqslant \frac{1}{4} \beta g^{2} .
$$

Hence (3.19) cannot be satisfied for $\beta g^{2} \leqslant 2 a_{1}$. On the other hand, if $P$ has no harmonic term, $a_{1}=0$, then $P^{\prime}\left(w^{2}\right)$ is monotone increasing from 0 to $\infty$. At the same time the right-hand side of (3.19) is a positive monotone decreasing function of $w^{2}$ and (3.19) has therefore a unique solution, $w_{0} \neq 0$, for any temperature.

We discuss the case of zero temperature, in the infinite volume limit $|\Lambda| \rightarrow \infty$, where the spectrum $e(k)^{2}$ of $T^{2}$ plays a fundamental role. In the infinite volume limit (3.20) reduces to

$$
P^{\prime}\left(w_{0}^{2}\right)=\frac{g^{2}}{2} \int_{[-\pi, \pi]^{d}} d^{d} k \frac{1}{\sqrt{e(k)^{2}+g^{2} w_{0}^{2}}} .
$$

If $P$ has no harmonic term, $a_{1}=0, P^{\prime}\left(w^{2}\right)$ is monotone increasing from 0 to $\infty$ while the righthand side of (3.22) is positive and monotone decreasing. Therefore (3.22) has always a unique solution $w_{0} \neq 0$. On the other hand, if $P$ has a harmonic term $a_{1}>0$, then $P^{\prime}\left(w^{2}\right) \geqslant a_{1}$, so for $g$ small enough (3.22) will not have a solution unless the integral diverges when $g \rightarrow 0$. Whether the integral diverges depends on the geometry of the lattice and the flux configuration:

(a) One-dimensional ring: $e(k)^{2}=(\cos k)^{2}$ so that the integral diverges and (3.22) has a solution $w_{0} \neq 0$. Here the flux plays no role.

(b) Cubical lattice with canonical flux: $e(k)^{2}=\sum_{i=1}^{d}\left(\cos k_{i}\right)^{2}$, which vanishes for the points $\left(k_{i}=\pi / 2, i=1, \ldots, d\right)$ and therefore the integral in (3.22) is convergent even for $g \rightarrow 0$. Therefore there exist $g_{c}>0$ such that, for $g \leqslant g_{c},(3.22)$ has no solution and $w_{0}=0$, and for $g \geqslant g_{c}$, (3.22) has a unique solution $w_{0} \neq 0$.

(c) Hexagonal lattice in $d=2$ with canonical flux: $e(k)^{2}$ vanishes only at isolated points and the situation is analogous to $(b)$.

For $\mu=\mathbf{h}=0$ at zero temperature the energy levels $e(k)<0$ are filled and those for which $e(k)>0$ are empty. The equation determining the Fermi surface is $e(k)=0$. In cases (b) and (c) 
where $d \geqslant 2$ the Fermi surface consists of isolated points, and as a consequence $w_{0}=0$ for $g$ small enough. In the context of the Holstein model this means that there is no Peierls instability for $g<g_{c}$.

When the flux is noncanonical and equal to zero the Fermi surface of the half-filled system, on a cubical lattice, is square shaped (see next paragraph) and this leads to a nonzero minimizer for all $g$.

Case of noncanonical flux configuration: When the flux configuration is not canonical, one cannot rely on Theorem 1 and other methods have to be used. The main example of such a case is the cubical lattice with $t_{x y}=t$ (zero flux). We rely on an inequality [(3.23) below] that was first derived in the context of the Falicov-Kimball model. ${ }^{7}$

We just require the lattice $\Lambda$ to be bipartite and for the moment no other specific hypothesis is made on $\left\{t_{x y}\right\}$. Then multiplication by $\epsilon_{x}$ is a unitary transformation such that $T \rightarrow-T$. Thus $E(\Phi)=E(-\Phi)$ and concavity of the square root implies

$$
E(\Phi)=\frac{1}{2} E(\Phi)+\frac{1}{2} E(-\Phi) \geqslant-\operatorname{tr} \sqrt{T^{2}+g^{2} \boldsymbol{\Phi}^{2}}+\sum_{x \in \Lambda} P\left(|\boldsymbol{\phi}(x)|^{2}\right),
$$

where $\boldsymbol{\Phi}^{2}$ is the $\Lambda \times \Lambda$ matrix with elements $\left.\boldsymbol{\phi}(x)\right|^{2} \delta_{x y}$. By expanding $h^{2}$ one checks that the equality is realized for Néel configurations (3.1). Now we proceed to analyze the consequences of this inequality.

Kondo model A: There $|\boldsymbol{\phi}(x)|^{2}=1$ so that the minimum is attained for configurations (3.1) with $w=1$. This result is valid for any flux configuration and is similar to what happens for the Falicov-Kimball model. ${ }^{8}$

Models $B, C$, and $D$ : In order to show that a Néel configuration is a minimizer of $E$, we have to check that the minimum of the rhs of (3.23) is itself a Néel configuration. The rhs of (3.23) is a convex functional of $\left\{\left|\boldsymbol{\phi}(x)^{2}\right|\right\}$ so that it has a unique minimizer $\left\{\left|\boldsymbol{\phi}_{0}(x)\right|^{2}\right\}$. It is given by the solution of the following set of $|\Lambda|$ equations

$$
P^{\prime}\left(\left|\boldsymbol{\phi}_{0}(x)\right|^{2}\right)=\frac{g^{2}}{2}\left\langle x\left|\left(T^{2}+g^{2} \boldsymbol{\Phi}_{0}^{2}\right)^{-1 / 2}\right| x\right\rangle, \quad x \in \Lambda,
$$

if such a solution exists (here we use the Dirac notation). If there is no such solution, then a minimum of (3.23) is attained on the boundary of the set $\left\{\left|\boldsymbol{\phi}_{0}(x)\right|^{2} \geqslant 0, x \in \Lambda\right\}$, in other words $\boldsymbol{\phi}_{0}(x)=0$ for at least one site $x \in \Lambda$.

Lemma: Suppose that $\left\{t_{x y}\right\}$ is such that the minimizer of the rhs of (3.23) is translation invariant, $\left|\boldsymbol{\phi}_{0}(x)\right|^{2}=w_{0}^{2}$, and $w_{0} \neq 0$. Then $E(\Phi)$ has a unique minimizer $\Phi_{0}(x)=\epsilon_{x} w_{0} \boldsymbol{\sigma} \cdot \hat{n}$.

Proof: From the hypothesis in the Lemma and (3.15)

$$
E(\Phi) \geqslant-\operatorname{tr} \sqrt{T^{2}+g^{2} w_{0}^{2} \mathbf{1}_{\Lambda}}+|\Lambda| P\left(w_{0}^{2}\right)=E\left(\Phi_{0}\right),
$$

and therefore $\Phi_{0}$ is a minimizer of $E$. Suppose that $E$ has a second minimizer $\Phi_{1} \neq \Phi_{0}$.

Then

$$
E\left(\Phi_{0}\right)=E\left(\Phi_{1}\right) \geqslant-\operatorname{tr} \sqrt{T^{2}+g^{2} \mathbf{\Phi}_{1}^{2} \mathbf{1}_{\Lambda}}+\sum_{x \in \Lambda} P\left(\left|\boldsymbol{\phi}_{1}(x)^{2}\right|\right)
$$

so that $\left|\boldsymbol{\phi}_{1}(x)\right|^{2}$ is also a minimizer for the rhs of (3.23). However, this is not possible since the latter has a unique minimum by convexity.

As a concrete example let us consider the case of a square lattice with $\left|t_{x y}\right|=t$, and a flux configuration equal to zero for all square plaquettes. Then it can be seen that the set of equations (3.22) has a uniform solution $\left|\boldsymbol{\phi}_{0}(x)\right|^{2}=w_{0}^{2}, w_{0} \neq 0$ for all $g \neq 0$. Indeed the spectrum of $T^{2}$ is 
$e(k)^{2}=\left(\sum_{i=1}^{d} \cos k_{i}\right)^{2}$, thus for $g \rightarrow 0$ the integral (3.22) has a logarithmic singularity due to the square shaped Fermi surface $e(k)=0$. We remark that the solution exists for all polynomials $P$.

For an arbitrary flux configuration the spectrum of $T$ is a very complicated set and we expect that the structure of the minimizers as a function of the flux is also more complicated, at least for models $\mathrm{B}$ and $\mathrm{C}$.

For the finite temperature functional $F(\Phi, \beta)$, the analog of inequality (3.23) is

$$
F(\Phi ; \beta) \geqslant-\frac{2}{\beta} \operatorname{tr} \ln \cosh \frac{\beta}{2} \sqrt{T^{2}+g^{2} \boldsymbol{\Phi}^{2}}+\sum_{x \in \Lambda} P\left(|\boldsymbol{\phi}(x)|^{2}\right) .
$$

Some consequences of (3.27) are the following:

Kondo model A: Equation (3.27) implies as before that the unique minimum of $F$ is (3.1) with $w=1$. This holds for an arbitrary bipartite lattice and any $\left\{t_{x y}\right\}$.

Models B, C, and D: The case of the Holstein model was analyzed in Ref. 11 on an infinite square lattice with $t_{x y}=t$. The results which also holds for models $\mathrm{C}$ and $\mathrm{D}$ are the following.

(i) If $P$ has no harmonic term, $a_{1}=0$, then for any $\beta$ and $g \neq 0, F$ attains its minimum for the Néel configurations, $\Phi_{0}(x)=\epsilon_{x} w_{0} \boldsymbol{\sigma} \cdot \hat{n}$ where $w_{0}^{2} \neq 0$ is the solution of the equation

$$
P^{\prime}\left(w_{0}^{2}\right)=\frac{g^{2}}{2} \int_{[-\pi, \pi]^{d}} d^{d} k E(k)^{-1} \tanh \left[\frac{\beta}{2} E(k)\right],
$$

with $E(k)=\left[4 t^{2}\left(\sum_{i=1}^{d} \cos ,{ }_{i}\right)^{2}+g^{2} w_{0}^{2}\right]^{1 / 2}$

(ii) If $P$ has a harmonic term, $a_{1}>0$, then given $g \neq 0$ there exist $\beta_{c}<\infty$ such that (3.28) has a solution $w_{0} \neq 0$ only for $\beta>\beta_{c}$. For $\beta>\beta_{c}$ the Néel configurations are the only minimizers, while for $\beta \leqslant \beta_{c}, \Phi_{0}(x)=0$ is the only minimizer.

We conclude this section with the following theorem.

Theorem 2: Let $\Lambda$ and $\left\{t_{x y}\right\}$ be arbitrary. Suppose that $P$ is a polynomial of order $N, N \geqslant 1$, all of whose coefficients are strictly positive, $a_{j}>0, j=1, \ldots, N$. Then for models $\mathrm{B}, \mathrm{C}$, and $\mathrm{D}$, there exists a positive number $c$ such that for $\beta g^{2}<c, F(\Phi ; \beta)$ is a strictly convex functional. Since it is even it attains its unique minimum at $\Phi(x)=0$, all $x \in \Lambda$.

The proof for the Holstein model can be found in Ref. 11 and also works for models C and D. A consequence of this theorem is the absence of long range order for $\beta g^{2}<c$ (see Sec. IV A).

\section{THERMAL AND QUANTUM FLUCTUATIONS}

In this section we address the question of stability of the ground states found in Sec. II when thermal or quantum fluctuations of the classical field are taken into account. Rigorous results have been obtained so far only for the Holstein model with $U=0$, and we will restrict ourselves to this case. We first consider thermal fluctuations alone, in two and three dimensions, and then quantum fluctuations at zero temperature for the one-dimensional system.

\section{A. Thermal fluctuations in $d=2,3$}

From (2.8) and (2.18) we have for the partition function

$$
Z_{\Lambda}(\beta, 0,0)=\int \prod_{x \in \Lambda} d \phi_{3}(x) \exp \left[-\frac{1}{2} \sum_{x \in \Lambda} \phi_{3}(x)^{2}+2 \operatorname{tr} \ln \cosh \frac{\beta}{2} \sqrt{\left(T+g \Phi_{3}\right)^{2}}\right] .
$$

In (4.1) $\Phi_{3}$ is the $|\Lambda| \times|\Lambda|$ matrix with elements $\phi_{3}(x) \delta_{x y}$, and the trace is over $l^{2}(|\Lambda|)$. The factor 2 in front of the trace comes from the spin degree of freedom and has no influence on the results (the spin becomes crucial when quantum fluctuations are taken into account, see Sec. IV B). From now on, we assume that the boundary conditions are periodic. As in Theorem 2 one can prove that 


$$
\frac{1-a}{2} \sum_{x \in \Lambda} \phi_{3}(x)^{2}+2 \operatorname{tr} \ln \cosh \frac{\beta}{2} \sqrt{\left(T+g \Phi_{3}\right)^{2}}
$$

is a convex functional of $\left\{\phi_{3}(x)\right\}$ for $\beta g^{2} \ll 1$ if $a<1$. It then follows from the Brascamp-Lieb inequalities that

$$
\left(\left\langle\phi_{3}(x) \phi_{3}(y)\right\rangle_{\Lambda}(\beta)\right)_{x, y \in \Lambda} \leqslant a^{-1 / 2} \mathbf{1}_{\Lambda}
$$

as quadratic forms. This implies that the Hilbert-Schmidt norm of the matrix on the left-hand side is bounded by $a^{-1}$ for all $|\Lambda|$,

$$
\frac{1}{|\Lambda|} \sum_{x, y \in \Lambda}\left|\left\langle\phi_{3}(x) \phi_{3}(y)\right\rangle_{\Lambda}(\beta)\right|^{2}<a^{-1},
$$

and clearly this is not compatible with the existence of long range order.

Remark: The same result can be proven also for the models C and D.

In situations where there are two antiferromagnetic ground states, it can be shown that there exist two corresponding low-temperature phases for a large enough coupling $g$. More precisely if $\Lambda$ is a square lattice with $\left|t_{x y}\right|=t$ and a uniform flux equal to $\pi$ or to 0 in all plaquettes, there exist a fixed number $\delta>0$ such that for $g$ and $\beta / g$ sufficiently large we have

$$
\pm w_{0}-\delta \leqslant\left\langle\epsilon_{x} \phi_{3}(x)\right\rangle^{ \pm}(\beta) \leqslant \pm w_{0}+\delta,
$$

where $w_{0}$ is the amplitude of the ground state, and $\langle-\rangle^{ \pm}(\beta)$ are expectations in the Gibbs states corresponding to the ordering on the different sublattices. They are obtained as infinite volume limits with appropriate boundary conditions.

The proof of (4.5) is based on a Peierls argument for continuous spins. ${ }^{11}$ The argument is quite involved because the "classical Hamiltonian" in (4.1) is not explicit, and relies on methods developed by Kennedy and $\mathrm{Lieb}^{7}$ for the case of the Falicov-Kimball model where $\phi_{3}(x)$ takes values \pm 1 , combined with an idea of Ref. 19 to take into account the "small" and "large" field configurations.

For the other three models discussed in this paper, the ground state breaks a continuous symmetry and therefore Peierls type arguments do not work. It is expected that there is long range order for dimensions greater or equal to three but a rigorous proof is lacking.

\section{B. Quantum fluctuations in one dimension}

The effect of quantum fluctuations has been analyzed ${ }^{12}$ so far only for the one-dimensional, spinless Holstein model with a dispersion term

$$
\begin{aligned}
H= & -\frac{t}{2} \sum_{x \in \Lambda}\left(c_{x}^{\dagger} c_{x+1}+c_{x+1}^{\dagger} c_{x}\right)+g \sum_{x \in \Lambda}\left(c_{x}^{\dagger} c_{x}-\frac{1}{2}\right) \phi_{3}(x) \\
& +\mu \sum_{x \in L} c_{x}^{\dagger} c_{x}+\sum_{x \in \Lambda}\left(-\frac{1}{2 m^{2}} \frac{\partial^{2}}{\partial \phi_{3}(x)^{2}}+\frac{1}{2} \phi_{3}(x)^{2}+b\left(\phi_{3}(x)-\phi_{3}(x+1)\right)^{2}\right) .
\end{aligned}
$$

It is shown in Ref. 12 that in the limit of zero temperature, for any fixed density of electrons $0<\rho<1$, and $g$ small enough (depending on $m, m^{-1} \neq 0$ ),

$$
\left\langle c_{x}^{\dagger} c_{y}\right\rangle \approx-\frac{\sin p_{F}(x-y)}{\pi|x-y|^{1+2 \eta(g)}}, \quad|x-y| \rightarrow \infty,
$$


with $p_{F}=\pi \rho$ and the anomalous exponent $\eta(g)$ analytic as a function of $g$. The exponent is nonvanishing as soon as $g \neq 0$ and the lowest nonvanishing order is $O\left(g^{6}\right)$ for $b=0$ and $O\left(g^{4}\right)$ for $b \neq 0$. Thus for $g \neq 0$ the electrons form a Luttinger liquid, and their momentum distribution has no jump discontinuity at $p=p_{F}$. Of course for $g=0$ the Fermi liquid behavior is recovered.

This behavior holds in particular for $\mu=0$ where $\rho=\frac{1}{2}$ and $p_{F}=\pi / 2$, and is completely different than in the case $m^{-1}=0$ (note that in the half-filled case one does not have to adjust the chemical potential as a function of $g$ to maintain $\rho=\frac{1}{2}$ ). Indeed when $m^{-1}=0$, a Peierls instability occurs and the ground state for the static phonons is $\phi_{3}(x)=\epsilon_{x} w_{0}, w_{0} \neq 0$ for all $g \neq 0$. Therefore the electrons see a period two potential and their spectrum is split into two bands, the lower one being filled, and the upper one empty so that (at zero temperature)

$$
\left\langle c_{x}^{\dagger} c_{y}\right\rangle \approx e^{-A(g)|x-y|}, \quad|x-y| \rightarrow \infty,
$$

where $A(g)$ is the energy gap separating the two bands at $p_{F}=\pi / 2$.

Thus as soon as $m^{-1} \neq 0$, for small enough coupling, the Peierls instability occuring at $\rho=\frac{1}{2}$ in the static case, disappears. We emphasize that these results are limited to small coupling and that at large enough coupling the Peierls instability is probably stable against the quantum fluctuations. ${ }^{20}$

Since the phonon field is harmonic and its coupling to the electrons is linear, one can easily integrate it out, and then one is left with a one-dimensional interacting fermionic system. In terms of Grassmanian anticommuting variables $\bar{\psi}(x, t), \psi(x, t),(x, t) \in \Lambda \times[0, \beta]$, the partition function becomes

$$
\begin{aligned}
Z_{\Lambda}(\beta, \mu)= & \int P_{\beta}(d \psi) \exp \left[-\mu \sum_{x \in \Lambda} \int_{0}^{\beta} \bar{\psi}(x, t) \psi(x, t)-\frac{g^{2}}{8} \sum_{x, y \in \Lambda} \int_{0}^{\beta} d s \int_{0}^{\beta} d t\right. \\
& \left.\times v(x-y, t-s)\left(\bar{\psi}(x, t) \psi(x, t)-\frac{1}{2}\right)\left(\bar{\psi}(y, s) \psi(y, s)-\frac{1}{2}\right)\right],
\end{aligned}
$$

where $P_{\beta}(d \psi)$ is the Grassmanian integral with propagator

$$
\frac{1}{\beta|\Lambda|} \sum_{e^{i k_{0} \beta=-1}} \sum_{e^{i k L}=1,|k|<\pi} \frac{e^{-i k_{0}(t-s)-i k(x-y)}}{-i k_{0}+\cos p_{F}-\cos k}
$$

and $v(x-y, t-s)$ is the effective potential between fermions that is induced by the phonons

$$
v(x-y, t-s)=\frac{1}{\beta|\Lambda|} \sum_{e^{i k_{0} \beta=1}} \frac{e^{i k_{0}(t-s)-i k(x-y)}}{m^{2} k^{2}+1+2 b^{2}(1-\cos k)} .
$$

For $m^{-1} \neq 0$ fixed, $b=0$ and $\beta, \Lambda \rightarrow \infty, v(x-y, t-s) \approx \delta_{x y}(2 m)^{-1} \exp \left(-m^{-1}|t-s|\right)$. We see that the effective interaction generated by the quantum fluctuations of the phonons is a two-body short range potential in the time direction and has zero range in the spatial direction. When $b \neq 0$ the situation is similar except that there is a more complicated short range interaction in the spatial direction. The behavior (4.7) is obtained by rigorous renormalization group methods developed in Ref. 21 for the one-dimensional spinless Fermi gas with short range interactions.

On the other hand, for $\beta, \Lambda$ fixed and $m^{-1} \rightarrow 0$, we get $v(x-y, t-s) \approx \beta^{-1}$, so that the effective interaction induced by static phonons is an infinite range potential of mean field type. In this situation the Luttinger liquid behavior breaks down and a Peierls instability occurs. 


\section{CONCLUSION: SOME OPEN PROBLEMS}

(i) In the static models one would like to analyze the low-temperature behavior when there is a continuous rotational symmetry (models $\mathrm{A}, \mathrm{C}$, and D). In situations where the ground states are of Néel type and break the symmetry, we expect that for dimensions greater or equal to three there is long range order. For example in the case of the static Kondo model with $U=0$, an expansion of (2.10) in powers of $g^{-1}$ for large $g$ gives, to first non-vanishing order, the classical Heisenberg Hamiltonian with a coupling constant proportional to $g^{-2}$. Therefore it is reasonable to expect long range order at large $\beta$ and $\beta g^{-2}$. However, in the case of the usual Heisenberg model the only known way to prove the existence of LRO is through the use of reflection positivity techniques, but here (unlike the case of zero temperature in Sec. III A) it is not clear how to proceed because the interaction is on-site. Analogous problems arise for the static approximations to the Hubbard model, where one also has to deal with the fact that the amplitude of the vector field is variable. We hope that the analysis of the Holstein model is a first step toward the solution of this problem.

(ii) We would like to understand the effect of spin, when quantum fluctuations are switched on. One can again perform the integration over the phonons and this leads to a two body potential which is attractive between spin up and down electrons. From the rigorous point of view the analysis of this case is still open even in one dimension. It is expected that in the half-filled band the ground state is ordered and has period two for all values of $g,{ }^{20}$ in contrast to the spinless case where this is not so for small $g$. An important problem is also the effect of quantum fluctuations in two or three dimensions; in this connection the techniques developed in Refs. 22 and 23 could be useful for the strong coupling case. Let us mention that Freericks and Lieb have proven that for the Holstein model on a connected finite lattice, when the Hamiltonian is real, for any even number of electrons, the ground state is unique and has zero total $\operatorname{spin}^{24}$ (see also Ref. 25 for a similar statement obtained previously by Lieb for the attractive Hubbard model).

(iii) A popular semi-quantum model, not covered by the present review, used to describe the polyacetylene chain, is the $\mathrm{Su}-\mathrm{Schrieffer-Heeger} \mathrm{model} \mathrm{(SSH).} \mathrm{There} \mathrm{the} \pi$-electrons are itinerant and hop on the chain, whereas the $\sigma$-electrons contribute to the effective elastic energy of the chain, which is modeled by a classical displacement field. The hopping amplitude of the $\pi$-electrons is a function of the displacements and this leads to an interaction between the classical and the quantum degrees of freedom. In order to investigate the effect of electron correlations one may also add a Hubbard interaction. For an extensive review of this model the reader can consult Ref. 26. It is proved in Ref. 27 and 18 that, in one dimension at half-filling, the ground state configuration of the displacements is either homogeneous or it has period two as predicted by the theory of the Peierls-Frohlich instability. Similar models and results have been discussed in two dimensions. ${ }^{28-30}$ In the SSH model it is expected, but there is no proof, that the Peierls instability persists when the quantum fluctuations of the positions of the atoms are taken into account. ${ }^{31}$

\section{ACKNOWLEDGMENTS}

N. M. wishes to thank Rutgers University for the hospitality he received while this work was completed.

This work was supported by NSF Grant No. DMR 92-13424.

\section{APPENDIX: THE STATIC APPROXIMATION}

We give some details on the derivation of the static approximations to the Hubbard models and show their relationship to the HF and BCS theories. For simplicity we consider $\mu=0, \mathbf{h}=0$.

Let us start with the repulsive model $(U>0)$ with Hamiltonian (2.2). Using the identity

$$
\left(c_{x \uparrow}^{\dagger} c_{x \uparrow}-\frac{1}{2}\right)\left(c_{x \downarrow}^{\dagger} c_{x \downarrow}-\frac{1}{2}\right)=-\frac{2}{3} \mathbf{S}(x)^{2}+\frac{1}{4},
$$


where $\mathbf{S}(x)=\frac{1}{2} \Sigma_{\alpha, \beta=\uparrow, \downarrow} c_{x \alpha}^{\dagger} \boldsymbol{\sigma}_{\alpha \beta} c_{x \beta}$ is the electron spin operator, the Hamiltonian becomes (up to a constant term)

$$
H_{\mathrm{elec}}=\sum_{x, y \in \Lambda} t_{x y} c_{x \sigma}^{\dagger} c_{y \sigma}-\frac{2 U}{3} \sum_{x \in \Lambda} \mathbf{S}(x)^{2}
$$

In terms of Grassman anticommuting variables for each spin component $\bar{\psi}_{\sigma}(x, t), \psi_{\sigma}(x, t)$ $(x, t) \in \Lambda \times[0, \beta]$, the partition function is given by ${ }^{13}$

$$
Z_{\text {Hubbard }}=\int P_{\beta}(d \psi) \exp \left[\frac{U}{6} \int_{0}^{\beta} d t \sum_{x \in \Lambda}\left(\sum_{\alpha, \beta=\uparrow, \downarrow} \bar{\psi}_{\alpha}(x, t) \boldsymbol{\sigma}_{\alpha \beta} \psi_{\beta}(x, t)\right)^{2}\right],
$$

where $P_{\beta}(d \psi)$ is the Grassmanian integral with the appropriate propagator [analogous to (4.10)]. The next step is an application of the Gaussian identity

$$
\begin{aligned}
& \int \prod_{i=1}^{3} \frac{d \phi_{i}(x, t)}{\sqrt{2 \pi}} \exp \left[-\frac{1}{2}|\boldsymbol{\phi}(x, t)|^{2}+\sqrt{\frac{U}{3}} \boldsymbol{\phi}(x, t) \cdot \sum_{\alpha, \beta=\uparrow, \downarrow} \bar{\psi}_{\alpha}(x, t) \boldsymbol{\sigma}_{\alpha \beta} \psi_{\beta}(x, t)\right] \\
& =\exp \left[\frac{U}{6}\left(\sum_{\alpha, \beta=\uparrow, \downarrow} \bar{\psi}_{\alpha}(x, t) \boldsymbol{\sigma}_{\alpha \beta}=\uparrow, \downarrow \psi_{\beta}(x, t)\right)^{2}\right],
\end{aligned}
$$

where $\boldsymbol{\phi}(x, t)$ is a time-dependent auxiliary field coupled to the electron spin. We get the formal expression

$$
Z_{\text {Hubbard }}=\int \mathscr{D} \boldsymbol{\phi} \exp \left[S_{\text {eff }}(\boldsymbol{\phi})\right]
$$

where $S_{\text {eff }}(\boldsymbol{\phi})$ is the effective action of the time-dependent field, and

$$
\begin{aligned}
\exp \left[S_{\text {eff }}(\boldsymbol{\phi})\right]= & \exp \left[-\frac{1}{2} \int_{0}^{\beta} d t \sum_{x \in \Lambda}|\boldsymbol{\phi}(x, t)|^{2}\right] \times \int P_{\beta}(d \psi) \\
& \times \exp \left[\sqrt{\frac{U}{3}} \int_{0}^{\beta} d t \sum_{x \in \Lambda} \boldsymbol{\phi}(x, t) \cdot \sum_{\alpha, \beta} \bar{\psi}_{\alpha}(x, t) \boldsymbol{\sigma}_{\alpha \beta} \psi_{\beta}(x, t)\right] .
\end{aligned}
$$

It is at this point that we make the static approximation. We replace the auxiliary field by a static one $\boldsymbol{\phi}(x)$. Then (A6) becomes equal to

$$
\begin{aligned}
& \exp \left[-\frac{\beta}{2} \sum_{x \in \Lambda}|\boldsymbol{\phi}(x)|^{2}\right] \int P_{\beta}(d \psi) \exp \left[\sqrt{\frac{U}{3}} \sum_{x \in \Lambda} \sum_{\alpha, \beta=\uparrow, \downarrow} \bar{\psi}_{\alpha}(x, t) \Phi_{\alpha \beta}(x) \psi_{\beta}(x, t)\right] \\
& =\operatorname{Tr} \exp \left[-\beta\left(H_{\mathrm{cin}}+H_{\text {int }}+\frac{1}{2} \sum_{x \in \Lambda}|\boldsymbol{\phi}(x)|^{2}\right)\right]=\exp [-\beta F(\Phi ; \beta)],
\end{aligned}
$$

where $H_{\text {int }}$ is given by (2.6) with $g=\sqrt{U / 3}$. We have thus obtained model (2.19)

To recover the HF mean field theory from the path integral formalism one evaluates the path integral $\mathscr{D} \boldsymbol{\phi}$ in (A5) by means of the saddle point approximation. The saddle point is the solution of

$$
\frac{\delta}{\delta \phi_{i}(x, t)} S_{\text {eff }}(\boldsymbol{\phi})=0, \quad i=1,2,3
$$


If one looks for static solutions of (A8), the equation reduces to

$$
\frac{\delta}{\delta \phi_{i}(x)} F(\Phi ; \beta)=0, \quad i=1,2,3,
$$

which yields (2.21). Therefore the Hartree-Fock approximation is equivalent to the variational problem of finding the global minimum of $F$.

In the case of the attractive model (2.2) with $U<0$, we use the identity

$$
\left(c_{x \uparrow}^{\dagger} c_{x \uparrow}-\frac{1}{2}\right)\left(c_{x \downarrow}^{\dagger} c_{x \downarrow}-\frac{1}{2}\right)=S_{1}^{\prime}(x)^{2}+S_{2}^{\prime}(x)^{2}-\frac{1}{4},
$$

where $S_{1}^{\prime}(x)$ and $S_{2}^{\prime}(x)$ are the components of the electron pseudospin operator

$$
S_{1}^{\prime}(x)=\frac{1}{2}\left(c_{x \uparrow}^{\dagger} c_{x \downarrow}^{\dagger}+c_{x \downarrow} c_{x \uparrow}\right), \quad S_{2}^{\prime}(x)=\frac{i}{2}\left(c_{x \uparrow}^{\dagger} c_{x \downarrow}^{\dagger}-c_{x \downarrow} c_{x \uparrow}\right)
$$

The Hamiltonian becomes up to a constant term

$$
H_{\text {elec }}=\sum_{x, y \in \Lambda} t_{x y} c_{x \sigma}^{\dagger} c_{y \sigma}-|U| \sum_{x \in \Lambda}\left(S_{1}^{\prime}(x)^{2}+S_{2}^{\prime}(x)^{2}\right)
$$

We then proceed in a way similar to the repulsive case, by applying another Gaussian identity

$$
\begin{aligned}
& \exp \frac{|U|}{4}\left[\left[\bar{\psi}_{\uparrow}(x, t) \bar{\psi}_{\downarrow}(x, t)+\psi_{\downarrow}(x, t) \psi_{\uparrow}(x, t)\right]^{2}-\left[\bar{\psi}_{\uparrow}(x, t) \bar{\psi}_{\downarrow}(x, t)-\psi_{\downarrow}(x, t) \psi_{\uparrow}(x, t)\right]^{2}\right] \\
& =\int \frac{\Pi_{i=1}^{2} d \phi_{i}(x, t)}{\sqrt{2 \pi}} \exp \left[-\frac{1}{2}\left(\phi_{1}(x, t)^{2}-\phi_{2}(x, t)^{2}\right)+\sqrt{\frac{|U|}{2}} \bar{\psi}_{\uparrow}(x, t) \bar{\psi}_{\downarrow}(x, t)\left[\phi_{1}(x, t)\right.\right. \\
& \left.\left.\quad+i \phi_{2}(x, t)\right]+\sqrt{\frac{|U|}{2}} \psi_{\downarrow}(x, t) \psi_{\uparrow}(x, t)\left[\phi_{1}(x, t)-i \phi_{2}(x, t)\right]\right]
\end{aligned}
$$

where $\phi_{1}(x, t), \phi_{2}(x, t)$ is a time-dependent auxiliary field coupled to the electron pseudospin. As before the static approximation consists in replacing it by a static classical field $\phi_{1}(x), \phi_{2}(x)$ which then gives (2.22).

\footnotetext{
${ }^{1}$ L. M. Falicov and J. C. Kimball, Phys. Rev. Lett. 22, 957 (1967).

${ }^{2}$ C. Gruber and N. Macris, Helv. Phys. Acta 69, 850 (1996).

${ }^{3}$ For a review of rigorous results on the true Hubbard model, see E. H. Lieb, "The Hubbard Model—Some Rigorous Results and Open Problems,"' in Proceedings of conference in honor of G. F. Dell'Antonio, Capri, Italy, 1993, Advances in dynamical systems and quantum physics, edited by S. Albeverio et al. (World Scientific, Singapore, 1995).

${ }^{4}$ T. Holstein, Ann Phys. 8, 325 (1959).

${ }^{5} \mathrm{~S}$. Aubry, in Microscopic aspects of Non linearity in condensed matter physics, edited A. R. Bishop, V. L. Pokrovsky, and V. Tognetti (Plenum, New York, 1991), p. 105-111.

${ }^{6}$ V. Bach, E. H. Lieb, and J. P. Solovej, J. Stat. Phys. 76, 3 (1994).

${ }^{7}$ T. Kennedy and E. H. Lieb, Physica 138, 320 (1986).

${ }^{8}$ E. H. Lieb and M. Loss, Duke. Math. J. 71, 337 (1993).

${ }^{9}$ E. H. Lieb, Phys. Rev. Lett. 73, 2158 (1994).

${ }^{10}$ N. Macris and B. Nachtergaele, J. Stat. Phys. 85, 745 (1996).

${ }^{11}$ J. L. Lebowitz and N. Macris, J. Stat. Phys. 76, 91 (1994).

${ }^{12}$ G. Benfatto, G. Gallavotti, and J. L. Lebowitz, Helv. Phys. Acta 68, 312 (1995).

${ }^{13}$ E. Fradkin, Field theories of condensed matter systems (Addison-Wesley, Reading, MA, 1991).

${ }^{14}$ S. Aubry, G. Abramovici, and J. L. Raimbault, J. Stat. Phys. 67, 675 (1992)

${ }^{15}$ C. Baesens and R. MacKay, J. Stat. Phys. (to appear).

${ }^{16}$ F. J. Dyson, E. H. Lieb, and B. Simon, J. Stat. Phys. 18, 335 (1978).

${ }^{17}$ J. Fröhlich, R. Israel, E. H. Lieb, and B. Simon, Commun. Math. Phys 62, 1 (1978).
} 
${ }^{18}$ E. H. Lieb, and B. Nachtergaele, Phys. Rev. B 51, 4777 (1995).

${ }^{19}$ G. Gallavotti and J. L. Lebowitz, J. Math. Phys. 12, 1129 (1971).

${ }^{20}$ E. Fradkin and J. E. Hirsch, Phys. Rev. B 27, 1680 (1983).

${ }^{21}$ G. Benfatto, G. Gallavotti, A. Procacia, and B. Scoppola, Commun. Math. Phys. 160, 93 (1994).

${ }^{22}$ A. Messager and S. Miracle-Solé, Rev. Math. Phys. 8, 271 (1996).

${ }^{23}$ N. Datta, R. Fernandez, and J. Fröhlich, J. Stat. Phys. 84, 455 (1996).

${ }^{24}$ J. Freericks and E. H. Lieb, Phys. Rev. B 51, 2812 (1995).

${ }^{25}$ E. H. Lieb, Phys. Rev. Lett. 62, 1201 (1989); Erratum 62, 1927 (1989).

${ }^{26}$ D. Baeriswyl, D. K. Campbell, and S. Mazumdar, in Conjugated conducting polymers, edited by H. Kiess, Springer Series in Solid State Sciences 102, 7 (1992).

${ }^{27}$ T. Kennedy and E. H. Lieb, Phys. Rev. Lett. 59, 1309 (1987).

${ }^{28}$ S. Tang and J. E. Hirsch, Phys. Rev. B 37, 9546 (1987).

${ }^{29}$ D. Baeriswyl and E. Jeckelmann, Mater. Sci. Forum 191, 71 (1995).

${ }^{30}$ N. Macris, Proceedings Karpacz Winter School, Poland, 1996, edited by Z. Petru et al. (Springer-Verlag, Berlin, 1997), Vol. 477.

${ }^{31}$ E. Fradkin and J. E. Hirsch, Phys. Rev. B 27, 4302 (1983). 